\title{
Grüneisen Parameter and Thermal Expansion by the Self-Consistent Renormalization Theory of Spin Fluctuations
}

\author{
Shinji Watanabe ${ }^{1}$ and Kazumasa Miyake ${ }^{2}$ \\ ${ }^{1}$ Department of Basic Sciences, Kyushu Institute of Technology, Kitakyushu, Fukuoka 804-8550, Japan \\ ${ }^{2}$ Center for Advanced High Magnetic Field Science, Osaka University, Toyonaka 560-0043, Japan
}

\begin{abstract}
The thermal expansion coefficient $\alpha$ and the Grüneisen parameter $\Gamma$ near the magnetic quantum critical point (QCP) are derived on the basis of the self-consistent renormalization (SCR) theory of spin fluctuation. From the SCR entropy, the specific heat $C_{V}, \alpha$, and $\Gamma$ are shown to be expressed in a simple form as $C_{V}=C_{\mathrm{a}}-C_{\mathrm{b}}, \alpha=\alpha_{\mathrm{a}}+\alpha_{\mathrm{b}}$, and $\Gamma=\Gamma_{\mathrm{a}}+\Gamma_{\mathrm{b}}$, respectively, where $C_{\mathrm{i}}, \alpha_{\mathrm{i}}$, and $\Gamma_{\mathrm{i}}(\mathrm{i}=\mathrm{a}, \mathrm{b})$ are related with each other. As the temperature $T$ decreases, $C_{\mathrm{a}}, \alpha_{\mathrm{b}}$, and $\Gamma_{\mathrm{b}}$ become dominant in $C_{V}, \alpha$, and $\Gamma$, respectively. The inverse susceptibility of spin fluctuation coupled to the volume $V$ in $\Gamma_{\mathrm{b}}$ is found to give rise to the divergence of $\Gamma$ at the QCP for each class of ferromagnetism and antiferromagnetism (AFM) in spatial dimensions $d=3$ and 2. This $V$-dependent inverse susceptibility in $\alpha_{b}$ and $\Gamma_{\mathrm{b}}$ contributes to the $T$ dependences of $\alpha$ and $\Gamma$, and even affects their criticality in the case of the AFM QCP in $d=2$. $\Gamma_{\mathrm{a}}$ is expressed as $\Gamma_{\mathrm{a}}(T=0)=-\frac{V}{T_{0}}\left(\frac{\partial T_{0}}{\partial V}\right)_{T=0}$ with $T_{0}$ being the characteristic temperature of spin fluctuation, which has an enhanced value in heavy electron systems.
\end{abstract}

\section{Introduction}

Quantum critical phenomena in itinerant electron systems have attracted considerable attention in condensed matter physics. When the continuous transition temperature of the magnetically ordered phase is suppressed to absolute zero by changing control parameters such as pressure and magnetic field, the quantum critical point (QCP) is realized. Near the QCP, the enhanced spin fluctuation causes the non-Fermi liquid behavior in physical quantities, which is called quantum critical phenomenon.

The self-consistent renormalization (SCR) theory of spin fluctuations developed by Moriya and coworkers has succeeded in explaining not only the Curie-Weiss behavior but also the quantum critical behavior in the magnetic susceptibility in the case of ferromagnetic criticality. ${ }^{1)}$ The SCR theory has also explained the quantum criticality in other physical quantities such as resistivity, specific heat, and NMR relaxation rate in the ferromagnetic and antiferromagnetic cases, ${ }^{2-6)}$ which has been endorsed by the renormalization group (RG) analysis by Hertz ${ }^{7)}$ and Millis. ${ }^{8)}$

The magneto-volume effect in nearly ferromagnetic (FM) metals $^{9)}$ and in antiferromagnetic (AFM) metals ${ }^{5}$ ) has been discussed by Moriya and coworkers. Kambe et al. pointed out a possibility that the Grüneisen parameter $\Gamma^{10)}$ diverges at the QCP. ${ }^{11)}$ By using the scaling hypothesis and the RG theory, Zhu et al. evaluated the critical part of the thermal expansion coefficient and the Grüneisen parameter, and showed that $\Gamma$ diverges at the QCP. ${ }^{12,13)}$

In this paper, we derive the thermal expansion coefficient and the Grüneisen parameter near the magnetic QCP on the basis of the SCR theory. By using the Maxwell relation, we show that $\alpha(T)$ derived from the pressure derivative of the entropy has a much simpler form than that derived from the temperature derivative of the pressure by the spin fluctuation theory. ${ }^{14)}$ Our result makes it possible to clarify the origin of the divergence of $\Gamma$ at the QCP, explicitly showing that the inverse susceptibility of spin fluctuation coupled to the volume gives rise to the divergence of $\Gamma$ at the QCP. Numerical calculations of $\alpha(T)$ and $\Gamma(T)$ are also performed for the FM QCP and AFM QCP in three- and two-spatial dimensions, which reveal that the quantum critical behavior appears at a sufficiently lower temperature than the characteristic temperature of spin fluctuation. We find that the volume derivative of the mode-mode coupling of spin fluctuation contributes to the temperature dependences of $\alpha(T)$ and $\Gamma(T)$ and even affects the quantum criticality at the AFM QCP in the two-spatial dimension.

This paper is organized as follows. In Sect. 2, the SCR theory is outlined and the nature of the specific heat near the QCP is summarized. The thermal expansion coefficient and the Grüneisen parameter are analyzed in Sects. 3 and 4, respectively. Comparison with experiments is discussed in Sect. 5. The paper is summarized in Sect. 6 .

\section{SCR Theory}

The SCR theory of spin fluctuations is outlined. First, the formalism of the SCR theory is explained in Sect. 2.1. In Sect. 2.2, the critical properties of the specific heat derived from the entropy are summarized. Hereafter, the energy units are taken as $\hbar=1$ and $k_{\mathrm{B}}=1$.

\subsection{Formalism of the SCR theory}

The action of interacting electrons is given in the form of the Ginzburg-Landau-Wilson functional

$$
\Phi[\varphi]=\frac{1}{2} \sum_{\bar{q}} \Omega_{2}(\bar{q}) \varphi(\bar{q}) \varphi(-\bar{q})
$$


$+\sum_{\bar{q}_{1}, \bar{q}_{2}, \bar{q}_{3}, \bar{q}_{4}} \Omega_{4}\left(\bar{q}_{1}, \bar{q}_{2}, \bar{q}_{3}, \bar{q}_{4}\right) \varphi\left(\bar{q}_{1}\right) \varphi\left(\bar{q}_{2}\right) \varphi\left(\bar{q}_{3}\right) \varphi\left(\bar{q}_{4}\right) \delta\left(\sum_{i=1}^{4} \bar{q}_{i}\right)$,

which has been derived from the Hamiltonian via the Hubbard-Stratonovich transformation applied to the onsite Coulomb interaction. ${ }^{7)}$ Here, $\bar{q}$ is the abbreviation for $\bar{q} \equiv$ $\left(\mathbf{q}, \mathrm{i} \omega_{l}\right)$, where $\omega_{l}=2 \pi l T(l=0, \pm 1, \pm 2, \cdots)$ is the Matsubara frequency with $T$ being the temperature. Note that Eq. (1) has the form derived from a single component of quadratic spin interaction (e.g., $\left.S_{i}^{z} S_{i}^{z}\right){ }^{7)}$ In the case of the isotropic Heisenberg interaction (e.g., $\mathbf{S}_{i} \cdot \mathbf{S}_{i}$ ), the factor 3 is to be multiplied to the right-hand side of Eq. (1). ${ }^{6,15,16)}$ In critical phenomena, long wavelength $|\mathbf{q}| \ll q_{\mathrm{c}}$ around the magnetically ordered vector $\mathbf{Q}$ and the low-frequency $|\omega| \ll \omega_{\text {c }}$ regions play dominant roles with $q_{\mathrm{c}}$ and $\omega_{\mathrm{c}}$ being the cutoffs for the momentum and frequency, respectively. Hence, the coefficients $\Omega_{i}$ for $i=2,4$ in Eq. (1) are expanded for $q$ and $\omega$ around $(\mathbf{Q}, 0)$ :

$$
\Omega_{2}\left(\mathbf{q}, \mathrm{i} \omega_{l}\right) \approx \frac{\eta_{0}+A q^{2}+C_{q}\left|\omega_{l}\right|}{N_{\mathrm{F}}}
$$

where $C_{q}$ is defined by $C_{q} \equiv C / q^{z-2}$ with $z$ being the dynamical exponent (e.g., $z=3$ for FM and $z=2$ for AFM) and $N_{\mathrm{F}}$ is the density of states at the Fermi level, and $\Omega_{4}\left(\bar{q}_{1}, \bar{q}_{2}, \bar{q}_{3}, \bar{q}_{4}\right) \approx$ $v_{4} /(\beta N)$ with $\beta \equiv 1 / T$.

To construct the action for the best Gaussian taking into account the mode-mode coupling of spin fluctuations up to the fourth order in $\Phi[\varphi]$, we use Feynman's inequality ${ }^{17)}$ on the free energy:

$$
F \leq F_{\text {eff }}+T\left\langle\Phi-\Phi_{\text {eff }}\right\rangle_{\text {eff }} \equiv \tilde{F}(\eta) .
$$

Here, the effective action $\Phi_{\text {eff }}$ is given by

$$
\Phi_{\mathrm{eff}}[\varphi]=\frac{1}{2} \sum_{l} \sum_{q} \frac{\eta+A q^{2}+C_{q}\left|\omega_{l}\right|}{N_{\mathrm{F}}}\left|\varphi\left(q, \mathrm{i} \omega_{l}\right)\right|^{2},
$$

where $\eta$ includes the effect of the mode-mode coupling of spin fluctuations and parameterizes the closeness to the quantum criticality. By the optimal condition $d \tilde{F}(\eta) / d \eta=0$, the selfconsistent equation for $\eta$, i.e., the SCR equation is obtained. By introducing the scaled form as $y \equiv \eta /\left(A q_{\mathrm{B}}^{2}\right), x \equiv q / q_{\mathrm{B}}$, $x_{\mathrm{c}} \equiv q_{\mathrm{c}} / q_{\mathrm{B}}$, and $t \equiv T / T_{0}$, where $T_{0}$ is the characteristic temperature of spin fluctuation defined by

$$
T_{0} \equiv \frac{A q_{\mathrm{B}}^{2}}{2 \pi C_{q_{\mathrm{B}}}},
$$

and $q_{\mathrm{B}}$ is the wave number characterizing the Brillouin zone, the SCR equation in the $d$-dimensional system is expressed as

$$
y=y_{0}+\frac{d}{2} y_{1} \int_{0}^{x_{\mathrm{c}}} d x x^{d+z-3}\left\{\ln u-\frac{1}{2 u}-\psi(u)\right\}
$$

for $d+z>4^{2-4)}$ and

$$
y=y_{0}+\frac{y_{1}}{2}\left(y \ln y+d \int_{0}^{x_{\mathrm{c}}} d x x\left\{\ln u-\frac{1}{2 u}-\psi(u)\right\}\right)
$$

for $d+z=4$. $^{5)}$ Here, $y_{0}$ and $y_{1}$ are constants, $u$ is defined as $u \equiv x^{z-2}\left(y+x^{2}\right) / t$, and $\psi(u)$ is the digamma function. The
(1) solutions of Eqs. (6) and (7) at the QCP can be obtained by inputting $y_{0}=0$ with $y_{1}$ and the cutoff $x_{\mathrm{c}}$ being set as constant values, e.g., $y_{1}=1$ and $x_{\mathrm{c}}=1$. The low $-t$ behavior of $y$ for each class of the $\operatorname{FM}(z=3)$ and $\operatorname{AFM}(z=2)$ in $d=3$ and 2, respectively, is listed in the first column of Table I. Note that the criticality in each class coincides with that shown by the RG theory ${ }^{8)}$ including logarithmic corrections in $d=2$.

\subsection{Entropy and specific heat}

The entropy $S=-\left(\frac{\partial \tilde{F}}{\partial T}\right)_{V}$ is obtained by differentiating the free energy $\tilde{F}$ with respect to the temperature under a constant volume as ${ }^{16)}$

$$
\begin{aligned}
S & =-N d \int_{0}^{x_{\mathrm{c}}} d x x^{d-1}\left\{\ln \sqrt{2 \pi}-u+\left(u-\frac{1}{2}\right) \ln u-\ln \Gamma(u)\right\} \\
& +N d \int_{0}^{x_{\mathrm{c}}} d x x^{d-1} u\left\{\ln u-\frac{1}{2 u}-\psi(u)\right\},
\end{aligned}
$$

where $\Gamma(u)$ is the Gamma function.

By differentiating the entropy $S$ in Eq. (8) with respect to the temperature under a constant volume, ${ }^{6,16)}$ the specific heat is obtained as

$$
\begin{aligned}
C_{V} & =T\left(\frac{\partial S}{\partial T}\right)_{V}, \\
& =C_{\mathrm{a}}-C_{\mathrm{b}},
\end{aligned}
$$

where $C_{\mathrm{a}}$ and $C_{\mathrm{b}}$ are given by

$$
\begin{aligned}
C_{\mathrm{a}} & =-N d \int_{0}^{x_{\mathrm{c}}} d x x^{d-1} u^{2}\left\{\frac{1}{u}+\frac{1}{2 u^{2}}-\psi^{\prime}(u)\right\}, \\
C_{\mathrm{b}} & =\tilde{C}_{\mathrm{b}}\left(\frac{\partial y}{\partial t}\right)_{V},
\end{aligned}
$$

respectively. Here, $\psi^{\prime}(u)$ is the trigamma function and $\tilde{C}_{\mathrm{b}}$ is given by

$$
\tilde{C}_{\mathrm{b}}=-N d \int_{0}^{x_{\mathrm{c}}} d x x^{d+z-3} u\left\{\frac{1}{u}+\frac{1}{2 u^{2}}-\psi^{\prime}(u)\right\} .
$$

The derivative $\left(\frac{\partial y}{\partial t}\right)_{V}$ in Eq. (11) can be calculated explicitly, by differentiating the SCR equation [Eqs. (6) and (7)] with respect to the scaled temperature $t$ under a constant volume:

$$
\left(\frac{\partial y}{\partial t}\right)_{V}=\left\{\begin{array}{l}
\frac{\frac{y_{1}}{2 t} \tilde{C}_{\mathrm{b}} \frac{1}{N}}{1-\frac{d y_{1}}{2 t} M} \text { for } d+z>4, \\
\frac{\frac{y_{1}}{2 t} \tilde{C}_{\mathrm{b}} \frac{1}{N}}{1-\frac{y_{1}}{2}(\ln y+1)-\frac{d y_{1}}{2 t} M} \quad \text { for } d+z=4,
\end{array}\right.
$$

where $M$ is given by

$$
M=\int_{0}^{x_{\mathrm{c}}} d x x^{d+2 z-5}\left\{\frac{1}{u}+\frac{1}{2 u^{2}}-\psi^{\prime}(u)\right\} .
$$

The low- $t$ behavior of $C_{\mathrm{a}}$ and $\tilde{C}_{\mathrm{b}}$ at the QCP for each class is summarized in the second and third columns of Table I, ${ }^{2-4,16,19)}$ respectively. For each class, $C_{\mathrm{a}}$ dominates over $C_{\mathrm{b}}$ as $t$ decreases and hence the specific heat behaves as

$$
C_{V} \approx C_{\mathrm{a}}
$$




\begin{tabular}{l|cccc}
\hline class & $y$ & $C_{\mathrm{a}}$ & $\tilde{C}_{\mathrm{b}}$ & $C_{V}$ \\
\hline $3 \mathrm{~d} \mathrm{FM}$ & $t^{\frac{4}{3}}$ & $-t \ln t$ & $t^{\frac{4}{3}}$ & $-t \ln t$ \\
$3 \mathrm{~d}$ AFM & $t^{\frac{3}{2}}$ & const. $-t^{1 / 2}$ & $t^{\frac{3}{2}}$ & const. $-t^{1 / 2}$ \\
$2 \mathrm{~d} \mathrm{FM}$ & $-t \ln t$ & $t^{\frac{2}{3}}$ & $-t \ln t$ & $t^{\frac{2}{3}}$ \\
$2 \mathrm{~d} \mathrm{AFM}$ & $-\frac{t \ln (-\ln t)}{\ln t}$ & $-t \ln t$ & $t \ln (-\ln t)$ & $-t \ln t$ \\
\hline
\end{tabular}

Table I. Quantum criticality at the magnetic QCP for each class specified by $z=3(\mathrm{FM})$ and $z=2(\mathrm{AFM})$ in spatial dimension $d=3$ and 2. ${ }^{1,18}$ ) Temperature dependences of $C_{\mathrm{a}}, \tilde{C}_{\mathrm{b}}$, and $C_{V}$ at the QCP for $t \ll 1 .^{2-4,16,19)}$

for $t \ll 1$. Hence, the criticality of $C_{V}$ is the same as that of $C_{\mathrm{a}}$ (see the last column of Table I). The criticality of $C_{V}$ in each class coincides with the RG theory. ${ }^{12,20)}$

\section{Thermal Expansion Coefficient near the Magnetic QCP}

Thus far, the thermal expansion coefficient $\alpha$ near magnetic transitions has been discussed with the spin-fluctuation theory ${ }^{14)}$ on the basis of the expression

$$
\alpha \equiv \frac{1}{V}\left(\frac{\partial V}{\partial T}\right)_{P}=\kappa_{T}\left(\frac{\partial P}{\partial T}\right)_{V},
$$

where $\kappa_{T}$ is the isothermal compressibility defined as

$$
\kappa_{T} \equiv-\frac{1}{V}\left(\frac{\partial V}{\partial P}\right)_{T} .
$$

In this paper, we show that $\alpha$ can be expressed in a much simpler form, which enables us to capture the physical meaning. We start from the expression

$$
\alpha=-\frac{1}{V}\left(\frac{\partial S}{\partial P}\right)_{T}
$$

equivalent to Eq. (16), which is transformed via the Maxwell relation $(\partial V / \partial T)_{P}=-(\partial S / \partial P)_{T}$. By differentiating the SCR entropy $S$ given by Eq. (8) with respect to the pressure under a constant temperature, we obtain

$$
\left(\frac{\partial S}{\partial P}\right)_{T}=-\frac{C_{\mathrm{a}}}{T_{0}}\left(\frac{\partial T_{0}}{\partial P}\right)_{T}-\frac{\tilde{C}_{\mathrm{b}}}{t}\left(\frac{\partial y}{\partial P}\right)_{T},
$$

where $C_{\mathrm{a}}$ and $\tilde{C}_{\mathrm{b}}$ are given in Eqs. (10) and (12), respectively. Here, we assume that the cutoff $x_{\mathrm{c}}$ in the $x$ integral in Eq. (8) has no pressure dependence (and hence no volume dependence) since it has been supposed that the choice of the cutoff does not affect the low-energy physics in the SCR theory. ${ }^{1)}$ Then, we obtain the thermal expansion coefficient as

$$
\alpha=\alpha_{\mathrm{a}}+\alpha_{\mathrm{b}},
$$

where $\alpha_{\mathrm{a}}$ and $\alpha_{\mathrm{b}}$ are defined as

$$
\begin{aligned}
& \alpha_{\mathrm{a}} \equiv \frac{1}{V} \frac{C_{\mathrm{a}}}{T_{0}}\left(\frac{\partial T_{0}}{\partial P}\right)_{T}, \\
& \alpha_{\mathrm{b}} \equiv \frac{1}{V} \frac{\tilde{C}_{\mathrm{b}}}{t}\left(\frac{\partial y}{\partial P}\right)_{T},
\end{aligned}
$$

respectively. Here, $(\partial y / \partial P)_{T}$ can be calculated by differentiating the SCR equation [Eqs. (6) and (7)] with respect to the pressure under a constant temperature as

$\left(\frac{\partial y}{\partial P}\right)_{T}=\left\{\begin{array}{l}\frac{\left(\frac{\partial y_{0}}{\partial P}\right)_{T}+\left(\frac{\partial y_{1}}{\partial P}\right)_{T} \frac{d}{2} L-\frac{1}{T_{0}}\left(\frac{\partial T_{0}}{\partial P}\right)_{T} \tilde{C}_{\mathrm{b}} \frac{y_{1}}{2} \frac{1}{N}}{1-\frac{d y_{1}}{2 t} M} \quad(d+z>4), \\ \frac{\left(\frac{\partial y_{0}}{\partial P}\right)_{T}+\left(\frac{\partial y_{1}}{\partial P}\right)_{T}\left(\frac{d}{2} L+\frac{1}{2} y \ln y\right)-\frac{1}{T_{0}}\left(\frac{\partial T_{0}}{\partial P}\right)_{T} \tilde{C}_{\mathrm{b}} \frac{y_{1}}{2} \frac{1}{N}}{1-\frac{y_{1}}{2}(\ln y+1)-\frac{d y_{1}}{2 t} M} \quad(d+z=4),\end{array}\right.$

where $L$ is defined as

$$
L \equiv \int_{0}^{x_{\mathrm{c}}} d x x^{d+z-3}\left\{\ln u-\frac{1}{2 u}-\psi(u)\right\} .
$$

Equation (20) is one of the central results of this paper.

The procedure for calculating $\alpha(t)$ is as follows: First, we solve the SCR equation [Eq. (6) or (7)] by inputting $y_{0}=0$, which corresponds to the QCP, with setting $y_{1}=1$ and $x_{\mathrm{c}}=1$. Then, by using this solution $y(t), C_{\mathrm{a}}(t)$ and $\tilde{C}_{\mathrm{b}}(t)$ are calculated as in Eqs. (10) and (12), respectively. By inputting the solution $y(t)$ into Eq. (23) with setting $\left(\partial y_{0} / \partial P\right)_{T}=1,\left(\partial y_{1} / \partial P\right)_{T}=1$, and $\left(\partial T_{0} / \partial P\right)_{T} / T_{0}=1$ as representative values (the reason for this parameterization is explained below), $(\partial y / \partial P)_{T}$ is obtained by calculating the right-hand side of Eq. (23). Finally, we obtain $\alpha_{\mathrm{a}}(t)$ in Eq. (21) and $\alpha_{\mathrm{b}}(t)$ in Eq. (22), resulting in $\alpha(t)$ in Eq. (20).

Here, we note the unit and the parametrization of $\alpha$. When we input the value of $\left(\partial T_{0} / \partial P\right)_{T} / T_{0}$ in the unit of $\mathrm{GPa}^{-1}$ and the molar volume as $V$ in the unit of $\AA^{3}$ into Eq. (21), $\alpha_{\text {a }}$ can be expressed in the unit of $\mathrm{K}^{-1}$ as

$$
\alpha_{\mathrm{a}}=\frac{0.0138}{V} \frac{1}{T_{0}}\left(\frac{\partial T_{0}}{\partial P}\right)_{T} \times \frac{C_{\mathrm{a}}}{N} .
$$

When we input the value of $(\partial y / \partial P)_{T}$ in the unit of $\mathrm{GPa}^{-1}$ and $V$ in the unit of $\AA^{3}$ into Eq. (22), $\alpha_{\mathrm{b}}$ can be expressed in the unit of $\mathrm{K}^{-1}$ as

$$
\alpha_{\mathrm{b}}=\frac{0.0138}{V}\left(\frac{\partial y}{\partial P}\right)_{T} \times \frac{\tilde{C}_{\mathrm{b}}}{N t} .
$$

Hence, multiplying the numerical values of the underlined terms in Eqs. (25) and (26) for each material to the following results of $\alpha_{\mathrm{a}}$ and $\alpha_{\mathrm{b}}$, respectively, shown in Figs. 1(a)-1(d), one can make a direct comparison with experiments.

The results of the numerical calculations of $\alpha(t)$ for the input parameters mentioned above for each universality class are shown in Figs. 1(a)-1(d). Note that, in each class, $\alpha_{\mathrm{b}}$ be-

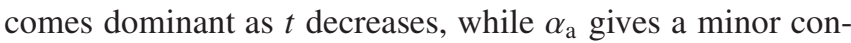
tribution.

For $d+z>4, \alpha$ behaves as

$$
\alpha \propto \frac{\tilde{C}_{\mathrm{b}}}{t}
$$

for $t \ll 1$, where $(\partial y / \partial P)_{T}$ is regarded as temperature independent, which is verified by the almost $t$-independent behavior shown in the inset of Figs. 1(a)-1(c). The critical behavior expressed as Eq. (27) actually appears for $t \ll 1$ as shown by the dashed line in Figs. 1(a)-1(c), which is summarized in the first column of Table II. The thermal expansion 

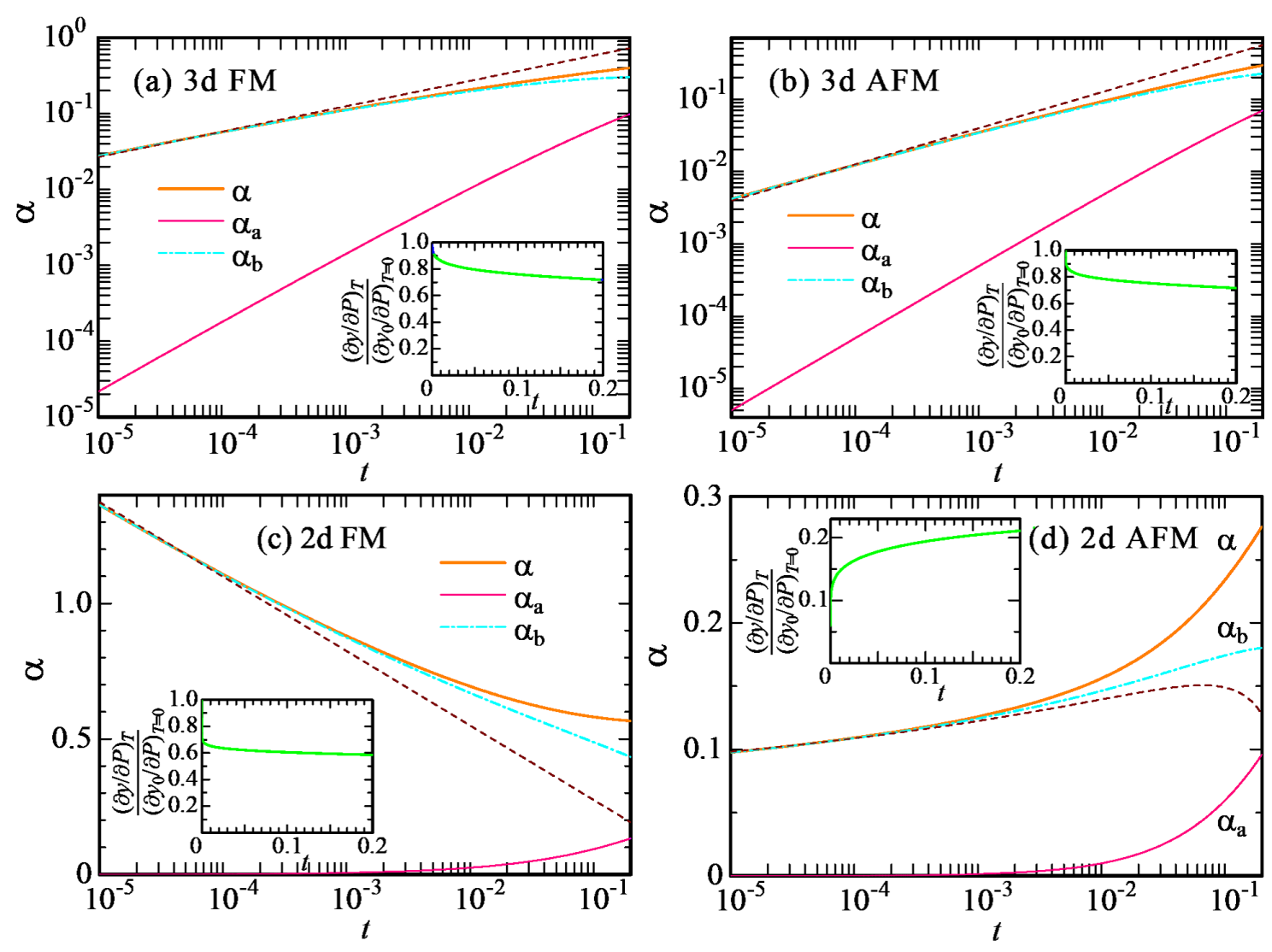

Fig. 1. (Color online) Thermal expansion coefficient $\alpha$ vs scaled temperature $t$ just at the QCP. The thick solid line is for $\alpha$, the thin solid line is for $\alpha_{\mathrm{a}}$, and the dashed-dotted line is for $\alpha_{\mathrm{b}}$. The dashed line represents the least-squares fit of $\alpha$ with $f_{\mathrm{i}}(t)(\mathrm{i}=\mathrm{a}, \mathrm{b}, \mathrm{c})$ for $10^{-5} \leq t \leq 10^{-4}$ and $f_{\mathrm{d}}(t)$ for $10^{-7} \leq t \leq 10^{-4}$. (a) 3d FM QCP: $f_{\mathrm{a}}(t)=a t^{1 / 3}$. (b) $3 \mathrm{~d}$ AFM QCP: $f_{\mathrm{b}}(t)=a t^{1 / 2}$. (c) $2 \mathrm{~d}$ FM QCP: $f_{\mathrm{c}}(t)=a \ln t$. (d) $2 \mathrm{~d}$ AFM QCP: $f_{\mathrm{d}}(t)=-a \frac{\ln (-\ln t)}{\ln \left(-\frac{t}{\ln t}\right)}$. The inset $\operatorname{shows}$ the $t$ dependence of $\left(\frac{\partial y}{\partial P}\right)_{T} /\left(\frac{\partial y_{0}}{\partial P}\right)_{T=0}$.

coefficient in the 2d FM case diverges $\alpha(t) \rightarrow \infty$ for $t \rightarrow 0$, whereas $\alpha(t) \rightarrow 0$ for $t \rightarrow 0$ in the $3 \mathrm{~d}$ FM and 3d AFM cases. These $t$ dependences coincide with those shown by the RG theory. ${ }^{12)}$ However, this asymptotic criticality appears only at sufficiently low temperatures for $t \lesssim 10^{-3}$ in the $3 \mathrm{~d}$ FM case [Fig. 1(a)] and the 3d AFM case [Fig. 1(b)], and for $t \lesssim 10^{-4}$ in the $2 \mathrm{~d} \mathrm{FM}$ case [Fig. 1(c)]. This is due to the presence of the weakly temperature-dependent $(\partial y / \partial P)_{T}$ in $\alpha_{\mathrm{b}}$, as shown in the inset of Figs. 1(a)-1(c).

For the marginal case $d+z=4$, i.e., the $2 \mathrm{~d}$ AFM case, $\alpha(t)$ for $t \ll 1$ is evaluated as

$$
\alpha \propto \frac{\tilde{C}_{\mathrm{b}}}{t}\left(\frac{\partial y}{\partial P}\right)_{T} \sim-\frac{\ln (-\ln t)}{\ln \left(-\frac{t}{\ln t}\right)} .
$$

This can be seen in Fig. 1(d), where $\alpha$ is well fit by the dashed line expressed as Eq. (28). Here, we found that $(\partial y / \partial P)_{T}$ has the temperature dependence even for $t \ll 1$ as $(\partial y / \partial P)_{T} \approx$ $\left(\partial y_{0} / \partial P\right)_{T=0}\left(-b_{4}\right) / \ln (-t / \ln t)$ with $b_{4}$ being a positive constant, which can be confirmed in the inset of Fig. 1(d). This is due to the logarithmic correction term in Eq. (7). Namely, $(\partial y / \partial P)_{T}$ affects the criticality in Eq. (28), which was not reported in the past RG studies. ${ }^{12,13)}$ Hence, the $t$ dependence

\begin{tabular}{l|cc}
\hline class & $\alpha$ & $\Gamma$ \\
\hline 3d FM & $t^{1 / 3}$ & $-\frac{t^{-2 / 3}}{\ln t}$ \\
3d AFM & $t^{1 / 2}$ & $\frac{t^{-1 / 2}}{\operatorname{const.~}}-\bar{r}^{1 / 2}$ \\
2d FM & $-\ln t$ & $-t^{-2 / 3} \ln t$ \\
2d AFM & $-\frac{\ln (-\ln t)}{\ln \left(-\frac{t}{\ln t}\right)}$ & $\frac{1}{t \ln t} \frac{\ln (-\ln t)}{\ln \left(-\frac{t}{\ln t}\right)}$ \\
\hline
\end{tabular}

Table II. Temperature dependences of $\alpha$ and $\Gamma$ at the QCP of FM $(z=3)$ and $\operatorname{AFM}(z=2)$ in spatial dimensions $(d=3,2)$.

of $\tilde{C}_{\mathrm{b}} / t$ showing divergence as $\sim \ln (-\ln t)$ for $t \rightarrow 0$ (see the third column in Table I), in agreement with the RG theory, ${ }^{12)}$ is counteracted by $(\partial y / \partial P)_{T} \rightarrow 0$ for $t \rightarrow 0$.

\section{Grüneisen Parameter near the Magnetic QCP}

The Grüneisen parameter $\Gamma$ is defined by

$$
\Gamma \equiv \frac{\alpha V}{C_{V} \kappa_{T}} .
$$

Since $\alpha$ is expressed as $\alpha_{\mathrm{a}}+\alpha_{\mathrm{b}}, \Gamma$ [Eq. (29)] is expressed as

$$
\Gamma=\Gamma_{\mathrm{a}}+\Gamma_{\mathrm{b}},
$$


where $\Gamma_{\mathrm{i}}(\mathrm{i}=\mathrm{a}, \mathrm{b})$ is defined by

$$
\Gamma_{\mathrm{i}} \equiv \frac{\alpha_{\mathrm{i}} V}{C_{V} \kappa_{T}} .
$$

At low temperatures, $C_{V}$ is governed by $C_{\mathrm{a}}$ as $C_{V}=C_{\mathrm{a}}-$ $C_{\mathrm{b}} \approx C_{\mathrm{a}}$. By using Eqs. (21) and (22), $\Gamma$ [Eq. (30)] is expressed for $t \ll 1$ as

$$
\begin{aligned}
\Gamma & \approx \frac{1}{\kappa_{T}} \frac{1}{T_{0}}\left(\frac{\partial T_{0}}{\partial P}\right)_{T}+\frac{\tilde{C}_{\mathrm{b}}}{C_{\mathrm{a}}} \frac{1}{t} \frac{1}{\kappa_{T}}\left(\frac{\partial y}{\partial P}\right)_{T}, \\
& =-\frac{V}{T_{0}}\left(\frac{\partial T_{0}}{\partial V}\right)_{T}-\frac{\tilde{C}_{\mathrm{b}}}{C_{\mathrm{a}}} \frac{V}{t}\left(\frac{\partial y}{\partial V}\right)_{T},
\end{aligned}
$$

where Eq. (17) has been used to derive the second line. One can see that the first and second terms of Eq. (32) correspond to $\Gamma_{\mathrm{a}}$ and $\Gamma_{\mathrm{b}}$, respectively.

Here, we note that Eq. (32) is consistent with $\Gamma$ derived under an adiabatic process. By differentiating both sides of Eq. (8) with respect to the volume under a constant entropy and using the expression $\Gamma=-\frac{V}{T}\left(\frac{\partial T}{\partial V}\right)_{S}$, which is equivalent to Eq. (29), we obtain

$$
\Gamma=-\frac{V}{T_{0}}\left(\frac{\partial T_{0}}{\partial V}\right)_{S}-\frac{\tilde{C}_{\mathrm{b}}}{C_{\mathrm{a}}} \frac{V}{t}\left(\frac{\partial y}{\partial V}\right)_{S} .
$$

We see that the first and second terms correspond to those in Eq. (32), respectively.

As for the first term in Eq. (32), $\Gamma_{\mathrm{a}}(T=0)=-\frac{V}{T_{0}}\left(\frac{\partial T_{0}}{\partial V}\right)_{T=0}$ is the volume derivative of the characteristic temperature of spin fluctuation. In heavy electron systems, $\left|\Gamma_{\mathrm{a}}\right|$ typically has an enhanced value with $O(10)$ being in the same order of the Grüneisen parameter in the Fermi-liquid regime $\Gamma_{\mathrm{FL}}$. Here, $\Gamma_{\mathrm{FL}}$ is defined as $\Gamma_{\mathrm{FL}} \equiv-\frac{V}{T_{\mathrm{K}}}\left(\frac{\partial T_{\mathrm{K}}}{\partial V}\right)_{T=0}$, where $T_{\mathrm{K}}$ is the characteristic temperature of heavy electrons called Kondo temperature. Since $\Gamma_{\mathrm{FL}}$ typically has an enhanced value ${ }^{21-23)}$ of $O(10)$ and $T_{0}$ is shown to be proportional to $T_{\mathrm{K}},\left|\Gamma_{\mathrm{a}}\right|$ is also enhanced although $\left|\Gamma_{\mathrm{a}}(t)\right|$ is almost $t$ independent.

To analyze the $t$ dependence of $\Gamma$ at the QCP, we performed the numerical calculation of Eq. (29). We calculate $\alpha$ by the procedure in Sect. 3. As for $C_{V}$, we calculate $C_{\mathrm{a}}$ and $C_{\mathrm{b}}$ in Eqs. (10) and (11), respectively, where $(\partial y / \partial t)_{V}$ is obtained by calculating Eq. (13). The input parameters set is the same as that set for Fig. 1. As for the isothermal compressibility, we confirmed that $\kappa_{T}$ does not show divergence even at the QCP for each class but has a finite value in general and hence we input $\kappa_{T}=0.1$ as a typical value for heavy electron systems. This is because $\frac{1}{T_{0}}\left(\frac{\partial T_{0}}{\partial P}\right)_{T}=\Gamma_{\mathrm{a}}(T=0) \kappa_{T}=1$ was used in Sect. 3 and $\kappa_{T}$ is set so as to reproduce $\Gamma_{\mathrm{a}}(T=0)=10$.

The results of the numerical calculations for each class are shown in Figs. 2(a)-2(d). Since $\Gamma_{\mathrm{a}}$ has a minor $t$ dependence here, we show the $t$ dependences of $\Gamma$ and $\Gamma_{\mathrm{b}}$. Reflecting the fact that $\alpha$ is dominated by $\alpha_{\mathrm{b}}$ for low $t$ (see Fig. 1), $\Gamma$ is mainly contributed from $\Gamma_{\mathrm{b}}$. Then, as $t$ decreases, $\Gamma$ increases and finally diverges for $t \rightarrow 0$ in each class because of the factor $1 / t$ in the last term of Eq. (32). Our analysis has revealed that the divergence of the Grüneisen parameter arises from the term with the volume derivative of the inverse susceptibility of spin fluctuation [see the last term of Eq. (32)].

For $d+z>4, \Gamma$ behaves as

$$
\Gamma \propto-\frac{\tilde{C}_{\mathrm{b}}}{C_{\mathrm{a}}} \frac{1}{t}
$$

for $t \ll 1$ because $(\partial y / \partial P)_{T}$ is almost $t$ independent as mentioned in Sect. 3. The critical $t$ dependence for each class, which can be known analytically from Table I, is actually confirmed by the numerical result well fitted by the dashed line in Figs. 2(a)-2(c). The criticality of $\Gamma$ is summarized in the second column of Table II, which is in agreement with the RG result. $^{12)}$ Note, however, that the criticality appears at a sufficiently low- $t$ regime for $t \lesssim 10^{-3}$, indicating that the temperature dependence of $(\partial y / \partial P)_{T}$ affects the intermediate- $t$ region.

For $d+z=4$, i.e., the $2 \mathrm{~d}$ AFM case, $\Gamma$ behaves as

$$
\Gamma \propto-\frac{\tilde{C}_{\mathrm{b}}}{C_{\mathrm{a}}} \frac{V}{t}\left(\frac{\partial y}{\partial V}\right)_{T} \sim \frac{1}{t \ln t} \frac{\ln (-\ln t)}{\ln \left(-\frac{t}{\ln t}\right)}
$$

for $t \ll 1$. The critical $t$ dependence comes from the multiplication of $\tilde{C}_{\mathrm{b}} /\left(C_{\mathrm{a}} t\right)$ (see Table I) and the prefactor $(\partial y / \partial P)_{T} \approx$ $1 / \ln (-t / \ln t)$, which can be confirmed numerically by the dashed line in Fig. 2(d). The $t$ dependence of $\tilde{C}_{\mathrm{b}} /\left(C_{\mathrm{a}} t\right)$ for $t \ll 1$ is in agreement with the RG theory. ${ }^{12)}$ Even after the inclusion of the $t$ dependence of $(\partial y / \partial P)_{T}, \Gamma$ diverges for $t \rightarrow 0$ since the factor $1 / t$ in $\tilde{C}_{\mathrm{b}} /\left(C_{\mathrm{a}} t\right)$ overcomes the logarithmic correction.

\section{Discussion}

To observe $\alpha(T)$ and $\Gamma(T)$ near the magnetic QCP, experimental measurements have been performed. ${ }^{11,24-28)}$ So far, a few data have been reported in stoichiometric compounds, which follow the criticality shown in Tables I and II.

$\mathrm{CeNi}_{2} \mathrm{Ge}_{2}$ at ambient pressure is regarded to be located closely to the $3 \mathrm{~d}$ AFM QCP since the low- $T$ data of the specific heat and resistivity show the $3 \mathrm{~d}-\mathrm{AFM}$ criticality in Table I. ${ }^{24)}$ The measured thermal expansion coefficient $\alpha=$ $c_{1} \sqrt{T}+c_{2} T$ is in agreement with $\alpha \sim T^{1 / 2}$ in Table I induced by spin fluctuation arising from the QCP and the FL contribution $\alpha_{\mathrm{FL}} \sim T$. The Grüneisen parameter $\Gamma \approx 57$ at $T=5 \mathrm{~K}$ is already enhanced because of the contribution from $\Gamma_{\mathrm{a}}$ and the heavy-electron background $\Gamma_{\mathrm{FL}}$. As $T$ decreases, $\Gamma$ further increases as $\Gamma \approx 98 \pm 10$ at $T \approx 0.1 \mathrm{~K}$, indicating the contribution from $\Gamma_{\mathrm{b}}$.

In $\mathrm{Ce}_{7} \mathrm{Ni}_{3}$, the $3 \mathrm{~d}$ AFM ordering is suppressed by applying pressure $P$ around $P_{\mathrm{c}}=0.39 \mathrm{GPa}$. As pressure increases, $T_{\mathrm{K}}(P)$ and $T_{0}(P)$ increase, and a smooth variation of both in the $T$ - $P$ phase diagram was observed. ${ }^{21,29)}$ This is understandable from the relation $T_{0} \propto T_{\mathrm{K}}$ as noted below Eq. (33). The measurements of $\alpha(T)$ and $\Gamma(T)$ at the QCP and their analyses based on Eqs. (20) and (30) are interesting studies left for the future.

\section{Summary}

On the basis of the SCR theory of spin fluctuations, we have derived the analytical expressions of the thermal expan- 

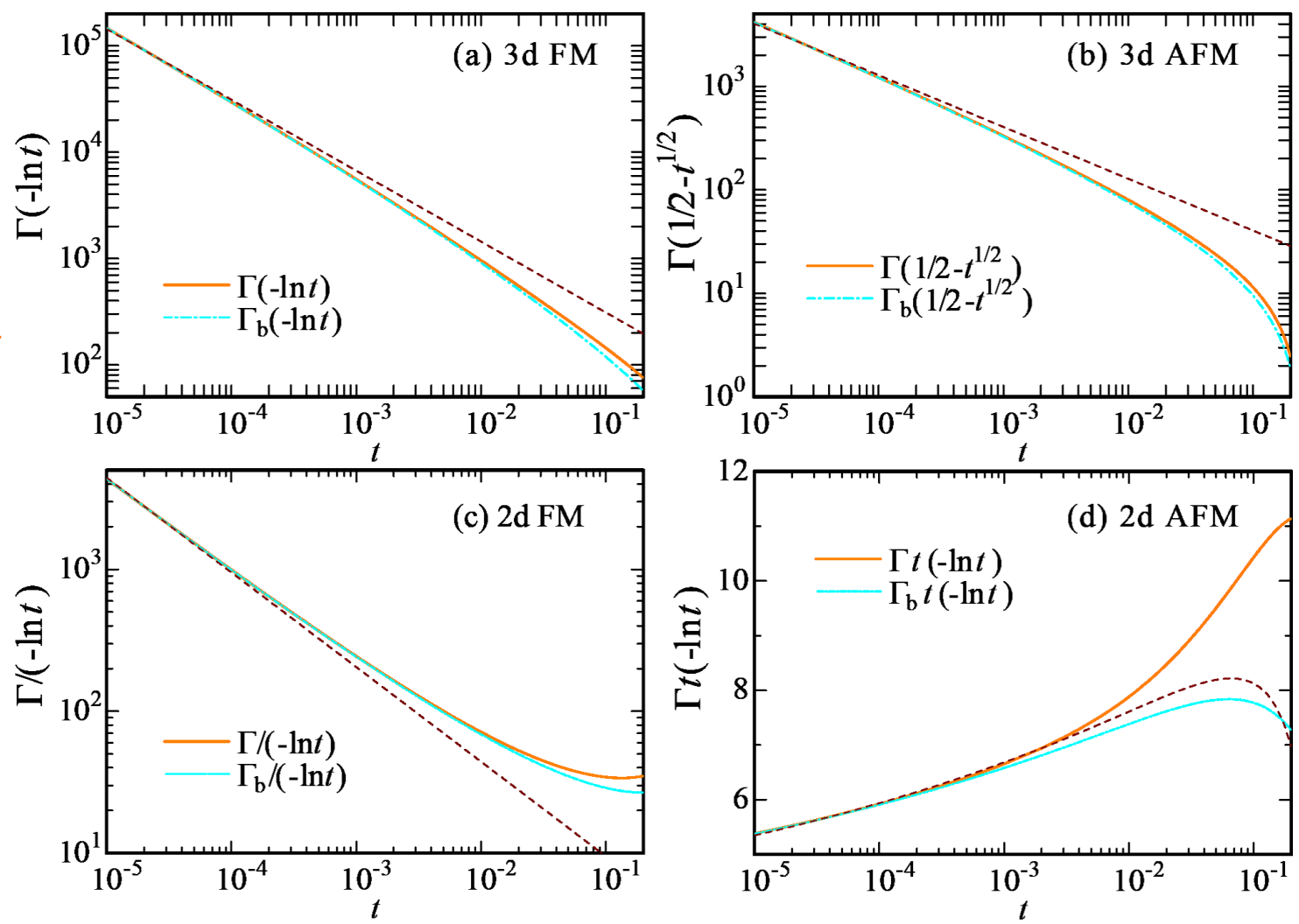

Fig. 2. (Color online) Temperature dependence of Grüneisen parameter $\Gamma$ (thick solid line) and $\Gamma_{\mathrm{b}}$ (dash-dotted line) just at the QCP. The dashed line represents the least-square fit of $\alpha$ with $g_{\mathrm{i}}(t)$ (i=a, b, c, d) for $10^{-5} \leq t \leq 10^{-4}$. (a) $3 \mathrm{~d} \mathrm{FM} \mathrm{QCP:} g_{\mathrm{a}}(t)=a t^{-2 / 3}$. (b) $3 \mathrm{~d}$ AFM QCP: $g_{\mathrm{b}}(t)=a t^{-1 / 2}$. (c) $2 \mathrm{~d}$ FM QCP: $g_{\mathrm{c}}(t)=a t^{-2 / 3}$. (d) $2 \mathrm{~d}$ AFM QCP: $g_{\mathrm{d}}(t)=-a \frac{\ln (-\ln t)}{\ln \left(-\frac{t}{\ln t}\right)}$.

sion coefficient and the Grüneisen parameter near the magnetic QCP and have numerically analyzed their properties.

The specific heat under a constant volume is expressed as $C_{V}=C_{\mathrm{a}}-C_{\mathrm{b}}$, where $C_{\mathrm{b}}$ has the form $C_{\mathrm{b}}=\tilde{C}_{\mathrm{b}}\left(\frac{\partial y}{\partial t}\right)_{V}$. We have derived the explicit forms of $\left(\frac{\partial y}{\partial t}\right)_{V}$ from the SCR equations for $d+z>4$ and $d+z=4$, respectively.

We have derived the thermal expansion coefficient from the expression of the SCR entropy through the relation $\alpha=$ $-(\partial S / \partial P)_{T} / V$ as $\alpha=\alpha_{\mathrm{a}}+\alpha_{\mathrm{b}}$ for each class, where $\alpha_{\mathrm{a}}=$ $\frac{1}{V} \frac{C_{\mathrm{a}}}{T_{0}}\left(\frac{\partial T_{0}}{\partial P}\right)_{T}$ and $\alpha_{\mathrm{b}}=\frac{\tilde{C}_{\mathrm{b}}}{t}\left(\frac{\partial y}{\partial P}\right)_{T}$. We have found that at low temperatures, $\alpha_{\mathrm{b}}$ dominates over $\alpha_{\mathrm{a}}$, while $C_{\mathrm{a}}$ dominates over $C_{\mathrm{b}}$ in each class. An important result is that there exists a temperature-dependent prefactor $(\partial y / \partial P)_{T}$ in $\alpha_{\mathrm{b}}$, which contributes to the intermediate-temperature region between the Curie-Weiss regime and quantum-critical regime. Furthermore, $(\partial y / \partial P)_{T}$ even affects the quantum criticality for $d+z=4$, i.e., the $2 \mathrm{~d}$ AFM case, giving rise to $\alpha(T) \rightarrow 0$ as $-\ln (-\ln t) / \ln (-t / \ln t)$, with $t=T / T_{0}$, for $T \rightarrow 0$.

On the basis of these correctly calculated $C_{V}$ and $\alpha$, we have derived the Grüneisen parameter. We have shown that $\Gamma$ is expressed as $\Gamma=\Gamma_{\mathrm{a}}+\Gamma_{\mathrm{b}}$, where $\Gamma_{\mathrm{i}}=\frac{\alpha_{i} V}{C_{V} K_{T}}(\mathrm{i}=\mathrm{a}, \mathrm{b})$. At low temperatures, $\Gamma_{\mathrm{a}}$ shows a minor $T$ dependence and is expressed as $\Gamma_{\mathrm{a}}=-\frac{V}{T_{0}}\left(\frac{\partial T_{0}}{\partial V}\right)_{T}$ for $T \rightarrow 0$, which has an enhanced value of typically $O(10)$ in the heavy electron systems. As temperature decreases, $\Gamma$ further increases, which is mainly contributed from $\Gamma_{\mathrm{b}} \approx-\frac{\tilde{C}_{\mathrm{b}}}{C_{\mathrm{a}}} \frac{V}{t}\left(\frac{\partial y}{\partial V}\right)_{T}$, and $\Gamma$ finally diverges for $T \rightarrow 0$ in each class. Our analysis has revealed that the divergence of the Grüneisen parameter arises from the inverse susceptibility of spin fluctuations coupled to the volume.

Numerical calculations of $\alpha(T)$ and $\Gamma(T)$ for each class show that the quantum-critical temperature dependence appears in the sufficiently low $T$ regime, which is typically below $T / T_{0} \lesssim 10^{-3}$ with $T_{0}$ being the characteristic temperature of spin fluctuation, owing to the $T$-dependent prefactor $(\partial y / \partial P)_{T}$. This is important when one makes a comparison with experiments.

\section{Acknowledgment}

The authors are grateful to $\mathrm{K}$. Umeo for discussions about experimental data of $\mathrm{Ce}_{7} \mathrm{Ni}_{3}$. This work was supported by Grants-in-Aid for Scientific Research (Grant Numbers JP24540378, JP25400369, JP15K05177, JP16H01077, and JP17K05555).

1) T. Moriya and A. Kawabata, J. Phys. Soc. Jpn. 34, 639 (1973); T. Moriya, Spin Fluctuations in Itinerant Electron Magnetism (Springer-Verlag, Berlin, 1985). 
2) M. Hatatani and T. Moriya, J. Phys. Soc. Jpn. 64, 3434 (1995).

3) T. Moriya and T. Takimoto, J. Phys. Soc. Jpn. 64, 960 (1995).

4) A. Ishigaki and T. Moriya, J. Phys. Soc. Jpn. 65, 376 (1996).

5) A. Ishigaki and T. Moriya, J. Phys. Soc. Jpn. 67, 3924 (1998).

6) A. Ishigaki and T. Moriya, J. Phys. Soc. Jpn. 68, 3673 (1999).

7) J. A. Hertz, Phys. Rev. B 14, 1165 (1976).

8) A. J. Millis, Phys. Rev. B 48, 7183 (1993); the statement "SCR procedure does not yield the $\log$ corrections in $d=2$ for $z=3$ and $z=2$." is not correct. The SCR results coincide with those derived by the renormalization group theory including the log corrections in $d=2$ for $z=3^{2)}$ and $z=2$ (T. Moriya: private communications).

9) T. Moriya and K. Usami, Solid State Commun. 34, 95 (1980).

10) E. Grüneisen, Ann. Phys. 39, 257 (1912).

11) S. Kambe, J. Flouquet, P. Lejay, P. Haen, and A. de Visser, J. Phys.: Condens. Matter 9, 4917 (1997).

12) L. Zhu, M. Garst, A. Rosch, and Q. Si, Phys. Rev. Lett. 91, 066404 (2003).

13) M. Garst and A. Rosch, Phys. Rev. B 72, 205129 (2005).

14) Y. Takahashi and H. Nakano, J. Phys.: Condens. Matter 18, 521 (2006).

15) The present results can be extended straightforwardly to the case of magnetic fluctuations of XY or Heisenberg type, where the factor of 2 or 3 is multiplied to the entropy in Eq. (8) and the specific heat in Eqs. (10) and (12). In real materials, anisotropy of the spin space more or less exists, yielding anisotropic spin fluctuation. Therefore, we plot $\alpha$ for a single component of the spin fluctuation with the factor of 1 in Fig. 1 for convenience of comparison with experiments. As for the Grüneisen parameter $\Gamma$, the results shown in Fig. 2 are not affected by this factor except for $\kappa_{T}$, since the factors in $\alpha$ and $C_{V}$ cancel each other in Eq. (31).

16) Y. Takahashi, J. Phys.: Condens. Matter 11, 6439 (1999).

17) R. P. Feynman, Statistical Mechanics (Addison-Wesley, Reading, Massachusetts, 1990) Sect. 3.4.

18) T. Moriya and K. Ueda, Rep. Prog. Phys. 66, 1299 (2003).

19) K. Makoshi and T. Moriya, J. Phys. Soc. Jpn. 38, 10 (1975).

20) U. Zülicke and A. J. Millis, Phys. Rev. B 51, 8996 (1995).

21) K. Umeo, H. Kadomatsu, and T. Takabatake, Phys. Rev. B 54, 1194 (1996).

22) J. Flouquet, Prog. Low. Temp. Phys. 15, 149 (2005).

23) A. V. Goltsev and M. M. Abd-Elmeguid, J. Phys.: Condens. Matter 17, 5813 (2005).

24) R. Küchler, N. Oeschler, P. Gegenwart, T. Cichorek, K. Neumaier, O. Tegus, C. Geibel, J. A. Mydosh, F. Steglich, L. Zhu, and Q. Si, Phys. Rev. Lett. 91, 066405 (2003).

25) R. Küchler, P. Gegenwart, J. Custers, O. Stockert, N. Caroca-Canales, C. Geibel, J. G. Sereni, and F. Steglich, Phys. Rev. Lett. 96, 256403 (2006).

26) R. Küchler, P. Gegenwart, C. Geibel, and F. Steglich, Sci. Technol. Adv Mater. 8, 428 (2007) and references therein.

27) A. Steppke, R. Kuchler, S. Lausberg, E. Lengyel, L. Steinke, R. Borth, T. Luhmann, C. Krellner, M. Nicklas, C. Geibel, F. Steglich, and M. Brando, Science 339, 933 (2013).

28) P. Gegenwart, Rep. Prog. Phys. 79, 114502 (2016) and references therein.

29) K. Umeo, H. Kadomatsu, and T. Takabatake, J. Phys.: Condens. Matter 8, 9743 (1996). 